\title{
LA CREATIVIDAD COMO MOTOR DE DESARROLLO. EL CASO DE SAN CRISTÓBAL DE LAS CASAS, CHIAPAS Y LA DECLARATORIA DE CIUDAD CREATIVA POR LA ARTESANÍA Y ARTE POPULAR DE LA UNESCO
}

\author{
CREATIVITY AS A DEVELOPMENT MOTIVATOR. \\ THE CASE OF SAN CRISTOBAL DE LAS CASAS, CHIAPAS, AND \\ THE DECLARATION OF CREATIVE CITY FOR THE CRAFTS AND \\ FOLK ART BY UNESCO
}

Mónica Solórzano Gil

Universidad Jesuita de Guadalajara, México

\section{RESUMEN}

Se presenta un análisis del potencial de la ciudad de San Cristóbal de Las Casas y la región Altos de Chiapas para aprovechar los diversos recursos e insumos creativos derivados de su riqueza y tradición cultural como uno de sus motores de desarrollo económico. Parte de los análisis que aquí se incluyen se derivan del proyecto de candidatura que desde 2013 impulsa la ciudad para integrarse a la Red de Ciudades Creativas por la Artesanía y Arte popular de la UNESCO ${ }^{1}$. En 2015 la ciudad es la primera en México en integrarse a esta red conformada por 19 ciudades en 15 países, 6 de ellas en América y el resto en Asia y Europa. Esto es un reto sin precedentes para México y pone de manifiesto la oportunidad de aprovechar su creatividad para generar cambios sustanciales en la calidad de vida de sus habitantes, aprovechando sus propios recursos de forma sustentable.

Palabras clave: Patrimonio cultural, creatividad, ciudades creativas, artesanía y arte popular, economía creativa.

Para mayor información consultar en http://sancristobalciudadcreativa.weebly.com/ 


\begin{abstract}
This paper presents an analysis of the potential of the city of San Cristobal de Las Casas and the Altos de Chiapas region to take advantage of the various resources and creative inputs derived from its rich cultural tradition as motivator of economic development. Part of the analysis included here is derived from the application project the city has promoted since 2013 in order to join the UNESCO Creative Cities Network of Craft and Folk $\mathrm{Art}^{1}$. In 2015 the city became the first in Mexico to join this network. This is an unprecedented challenge for Mexico and highlights the opportunity to use its creativity to generate substantial changes in the quality of life of its inhabitants using its own resources in a sustainable way.
\end{abstract}

Keywords: Cultural heritage, creativity, creative cities, crafts and folk art, creative economy.

\title{
Introducción
}

La ciudad de San Cristóbal de Las Casas se localiza en el sureste de México en el Estado de Chiapas, colinda con municipios con población indígena tseltal y tsotsil y limita hacia el sur del territorio con la frontera de Guatemala. Es considerada una de las ciudades más antiguas fundadas por los españoles en el sur de México en 1528 y cuenta desde su fundación con una estructura urbana particular, configurada por barrios, los cuales surgen con una vocación y oficio específico, o lo fueron adquiriendo al pasar del tiempo, y las necesidades materiales de la ciudad. Asimismo por su localización geográfica en la Región Altos de Chiapas² ${ }^{2}$ la ciudad ha estado estrechamente vinculada a los 17 municipios y a las comunidades indígenas rurales que en ella habitan, que a su vez cuentan con una importante actividad productiva en oficios y labores artesanales diversas.

En este contexto geográfico la ciudad de San Cristóbal de Las Casas ha tenido un papel neurálgico como nodo de actividad económica para la región, ya que históricamente y aún hoy en día, la población de estos municipios concurre en ella para el intercambio y abasto de bienes, productos y servicios, siendo esto uno de los factores importantes para la economía de la ciudad.

En contraste a toda esta riqueza cultural y creativa, en esta región de Chiapas se localizan 9 de los 15 municipios que, de acuerdo a indicadores oficiales del Consejo Nacional de Evaluación de la Política de Desarrollo Social (CONEVAL, 2011), esta zona presenta índices elevados de pobreza así como los menores niveles de desarrollo en México. Entonces, partiendo de esto toca preguntarse ¿Cómo aprovechar la riqueza cultural de estas comunidades para cambiar su realidad económica e incentivar el desarrollo económico capitalizando esta creatividad? Sin duda, este es el principal reto que se tiene y que analizaremos en este artículo.

2 El Estado de Chiapas se divide en varias regiones demográficas para facilitar su administración estatal para la aplicación de programas estatales y federales. La región $\vee$ Altos Tsotsil-Tseltal incluye 17 municipios en su mayoría indígenas. 
Figura 1. Vista de la Catedral de San Cristóbal de Las Casas, el edificio más emblemático de la ciudad.

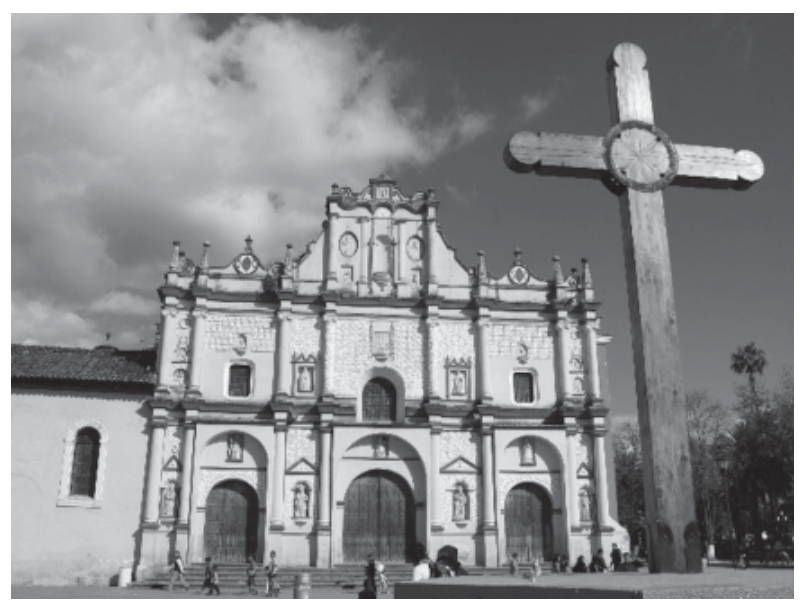

Fuente: Documento fotográfico de Mónica Solórzano
Figura 2. Calle típica de la ciudad con la presencia de artesanas comercializando sus productos.

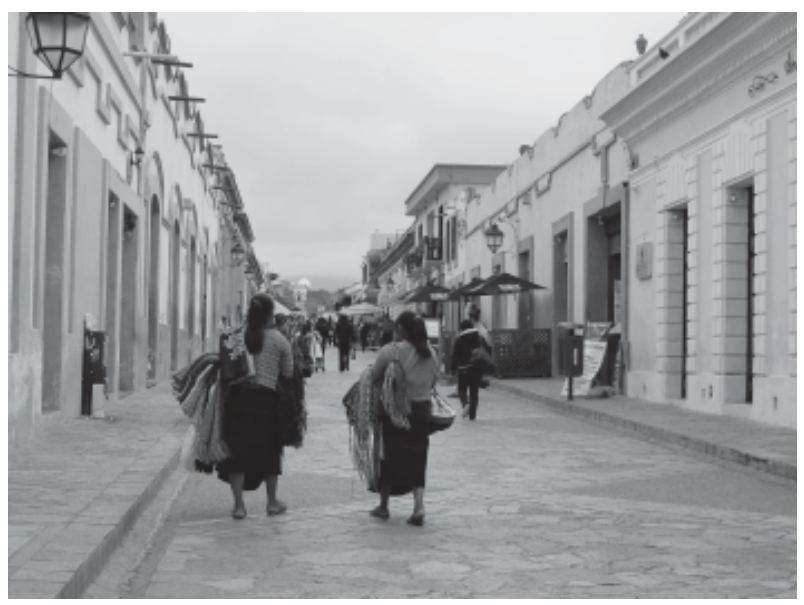

Fuente: Documento fotográfico de Mónica Solórzano

\section{Objetivos}

Tomando en cuenta esta riqueza y potencial y buscando una estrategia para aprovechar la creatividad con la que cuentan, este artículo aborda la estrategia en la que el Ayuntamiento Municipal de San Cristóbal de Las Casas en el año 2013 se propuso trabajar para integrarse a la Red de Ciudades Creativas de la UNESCO. El objetivo de esta intención de pertenecer a dicha Red es lograr la revaloración de su cultura en nivel local, nacional e internacional para impulsar y fortalecer las actividades creativas, como la base del modelo económico de desarrollo. Con esto, se pretende desde la instancia municipal alinear estrategias y políticas públicas para beneficiar directamente a los habitantes de la ciudad y las comunidades de la región, impulsando nuevos modelos y paradigmas de desarrollo económicos basados en su propia creatividad, para aprovechar su potencial para el fomento de su cultura y utilizándola como motor para impulsar su propio desarrollo.

Para llevar a cabo esta iniciativa de convertirse en Ciudad Creativa y apostar a este tipo de desarrollo en torno a la economía creativa, es preciso entender el concepto y lo que esto implica, ya que es aquella economía que abarca no sólo bienes y servicios culturales, sino también la que comprende los sectores en los que el valor de sus bienes y servicios se fundamenta en la propiedad intelectual. (Buitrago \& Duque, 2013, p. 15).

Asimismo, la economía creativa abarca sectores y procesos que tienen como insumo la creatividad, sobre todo la cultura, para generar en el ámbito local y distribuir en el ámbito global bienes y servicios con valor simbólico y económico (Buitrago \& Duque, 2013, p. 24).

Esto reconoce las actividades y los procesos culturales como el núcleo de una nueva y poderosa economía. Por lo tanto, las industrias creativas o culturales son aquellas que se enfocan en la producción y consumo 
cultural que tienen un elemento expresivo y simbólico en su núcleo y que abarcan campos y sectores creativos muy diversos, su alcance no se limita únicamente a la producción intensiva con base en la tecnología, sino que considera que una gran parte de la producción cultural de los países en desarrollo es de tipo artesanal.

La UNESCO (2015) a su vez define a las industrias creativas o culturales como: «aquellos sectores de actividad organizada que tienen como objeto principal la producción o la reproducción, la promoción, la difusión y/o la comercialización de bienes, servicios y actividades de contenido cultural, artístico o patrimonial».

Por consecuencia los recursos y beneficios económicos que se generan a partir de estos sectores creativos y estas actividades relacionadas a ellos dan cuerpo a la economía creativa de cada lugar, la cual se aprovecha de lo singular, lo simbólico y lo intangible (Fonseca, 2008, p. 9), como insumos para hacer más fuerte y apuntalar esta economía. Esto contribuye al reconocimiento, recuperación y puesta en valor de lo que se genera y produce en las ciudades de forma única y particular y al fomento y desarrollo de las industrias creativas, contribuyendo así a la preservación de la identidad cultural. (Solórzano, 2015, p. 160)

Como contexto general, la red de Ciudades Creativas por la Artesanía y Arte y Popular de la UNESCO, en la que San Cristóbal de Las Casas se integró en 2015, se ha ido configurando y creciendo de forma importante en los últimos años, integrando diversas ciudades de distintos países y regiones en el mundo. En 2016 la Red y está conformada de la siguiente forma:

Tabla 1

Configuración cronológica de la Red de ciudades creativas por la artesanía y arte popular de la UNESCO entre 2013 y 2015

Configuración de la Red de ciudades creativas por la artesanía y arte popular de la UNESCO

\begin{tabular}{|c|c|c|c|c|c|}
\hline \multicolumn{2}{|r|}{2013} & \multicolumn{2}{|r|}{2014} & \multicolumn{2}{|r|}{2015} \\
\hline 1. & Santa Fe (Estados Unidos) & 6. & Fabriano (Italia) & 10. & Nassau (Bahamas) \\
\hline 2. & Aswan (Egipto) & 7. & Paducah (Estados Unidos) & 11. & Pekalongan (Indonesia) \\
\hline 3. & Kanazawa (Japón) & 8. & Jacmel (Haiti) & 12. & Suzhou (China) \\
\hline 4. & Hangzhou (China) & 9. & Jingdezhen (China) & 13. & Al-Ahsa (Arabia Saudita) \\
\hline \multirow[t]{6}{*}{5.} & Icheon (Corea del Sur) & & & 14. & Bamiyan (Afghanistan) \\
\hline & & & & 15. & Durán (Ecuador) \\
\hline & & & & 16. & Isfahan (República Islámica de Irán) \\
\hline & & & & 17. & Lubumbashi (República Democrática del Congo) \\
\hline & & & & 18. & Sasayama (Japón) \\
\hline & & & & 19. & San Cristóbal de las Casas (México) \\
\hline
\end{tabular}

Fuente y elaboración propia 
Figura 3. Mapa que muestra la Red de Ciudades Creativas por la artesanía y arte popular de la UNESCO en 2015 y la localización de San Cristóbal de Las Casas, México.

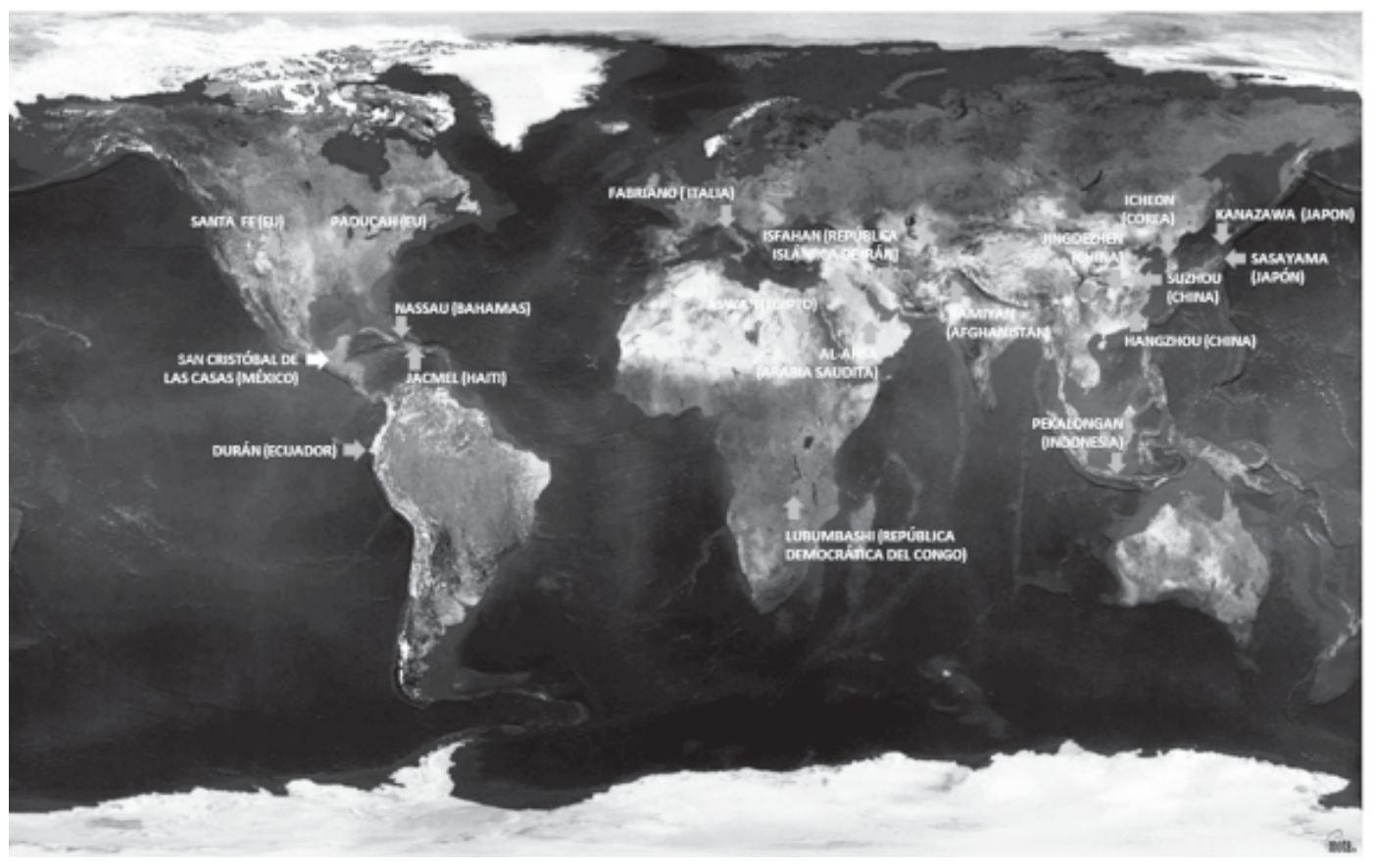

Fuente: Documento fotográfico de Mónica Solórzano

\section{Historia y contexto general de la Ciudad y la Región}

La ciudad de San Cristóbal de Las Casas y sus barrios tradicionales fueron configurándose durante cuatro siglos, en donde la iglesia era el eje de la vida económica, política, religiosa y social. Originalmente en la época colonial, la ciudad fundacional se componía principalmente de dos áreas, el recinto y el barrio. El recinto era el centro y abarcaba algunas cuadras alrededor de la plaza mayor, en las que habitaban familias españolas y gente a su servicio. Después del primer núcleo se crearon los primeros cinco barrios indígenas, fundados en 1528, que son: el barrio de Mexicanos, (conformado por indios Mexicas), el barrio de San Diego (conformado por indígenas Zapotecos), el barrio de San Antonio (conformado por indígenas Mixtecos) y el barrio de Cuxtitali (conformado por indígenas Quiches). Los primeros tres barrios estaban integrados por indígenas amigos que habían acompañado al conquistador Diego de Mazariegos desde la zona central de México.

Como se puede apreciar, históricamente la ciudad tiene un origen marcado por la migración, ya que desde la ocupación española fueron llegando junto a los conquistadores, culturas como la Mexica y los Tlaxcaltecas, agregándose posteriormente otros grupos étnicos que fueron ocupando la ciudad fundando los diferentes barrios indígenas que rodeaban al llamado «recinto» donde vivían los españoles. De esta forma, espacialmente la ciudad adquiere su característico y singular modelo urbano de ciudad «dual» (Aubry, 2008). 
Figura 4. Barrio de Cuxtitali.

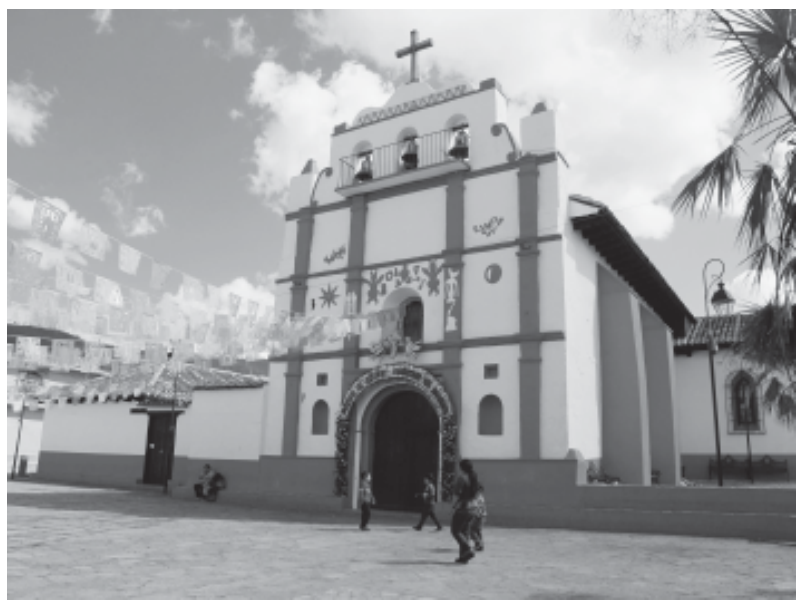

Fuente: Documento fotográfico de Mónica Solórzano
Figura 5. Barrio de Mexicanos.

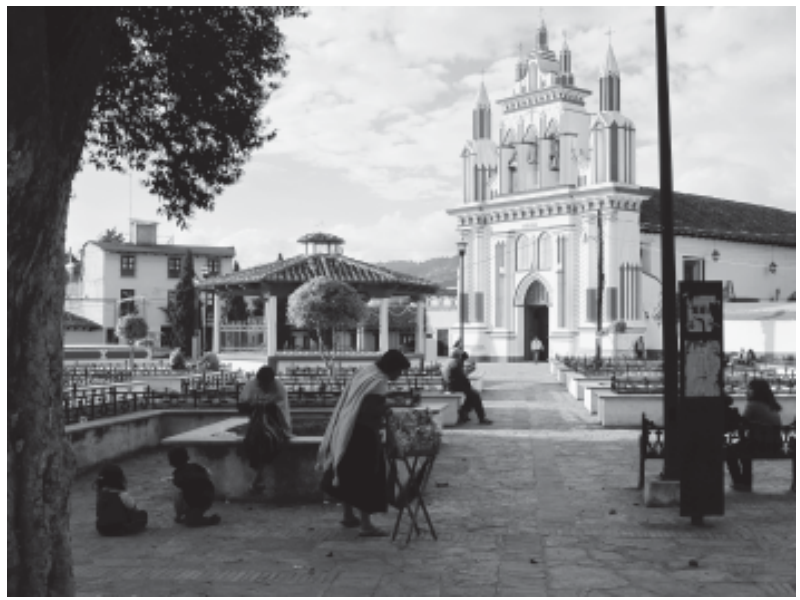

Fuente: Documento fotográfico de Mónica Solórzano

Actualmente San Cristóbal de Las Casas es un municipio urbano rural con población intercultural de aproximadamente 185917 habitantes y en donde existen diversos grupos indígenas que hablan lenguas como el tseltal y tsotsil. Asimismo que por su localización geográfica, la ciudad está relacionada con muchas poblaciones indígenas rurales provenientes de los 17 municipios de la «Región Altos de Chiapas». La población de estos municipios acude a la ciudad para el intercambio y abasto de bienes, productos y servicios, que se comercializan en sus calles, plazuelas, mercados, comercios diversos y se puede encontrar a los indígenas artesanos que ofrecen una amplia variedad de artesanías y textiles típicos y productos de sus comunidades. Esto la convierte en un centro de reunión de culturas vivas único en el mundo. Además de esto, la ciudad cuenta con una amplia infraestructura cultural, sumado a la riqueza arquitectónica y paisajes naturales.

\section{Insumos creativos de la Ciudad y la Región}

Para lograr visualizar el potencial de la cultura y la creatividad como impulsores de cambio y desarrollo económico se analizan, en este artículo, cuáles son los diversos insumos y recursos con que se cuenta en los ámbitos local y regional. El ámbito local considera la ciudad y sus barrios tradicionales así como las comunidades del municipio vinculadas a ella, mientras el ámbito regional considera los municipios y comunidades de la Región Altos de Chiapas. Un tercer ámbito considera la relación que deberá establecer la ciudad de San Cristóbal de Las Casas en el plano internacional con las ciudades que ya forman parte de la Red de Ciudades Creativas de la UNESCO. Asimismo, en este ámbito se incluyen las ciudades hermanas, con las cuales, la ciudad ya tiene firmados acuerdos de cooperación internacional y que contribuyen a la vinculación de la ciudad en este contexto.

En cuanto a los insumos creativos, en el primer ámbito de lo local encontramos que en los barrios tradicionales de San Cristóbal se elaboran oficios diversos como la alfarería, carpintería, talabartería, la gastronomía tradicional que incluye panadería, dulces y platos típicos elaborados con productos locales; la cerería 
o fabricación de velas y veladoras artesanales; así como la fabricación de hilados y tejidos; y el teñido y bordado textil, entre otros. En este mismo ámbito se consideran las diversas comunidades del municipio que también desarrollan actividades productivas en oficios diversos, además de aquellos propios que producen los barrios tradicionales de la ciudad.

Figura 6. Ámbito Local. Los barrios tradicionales de la ciudad.

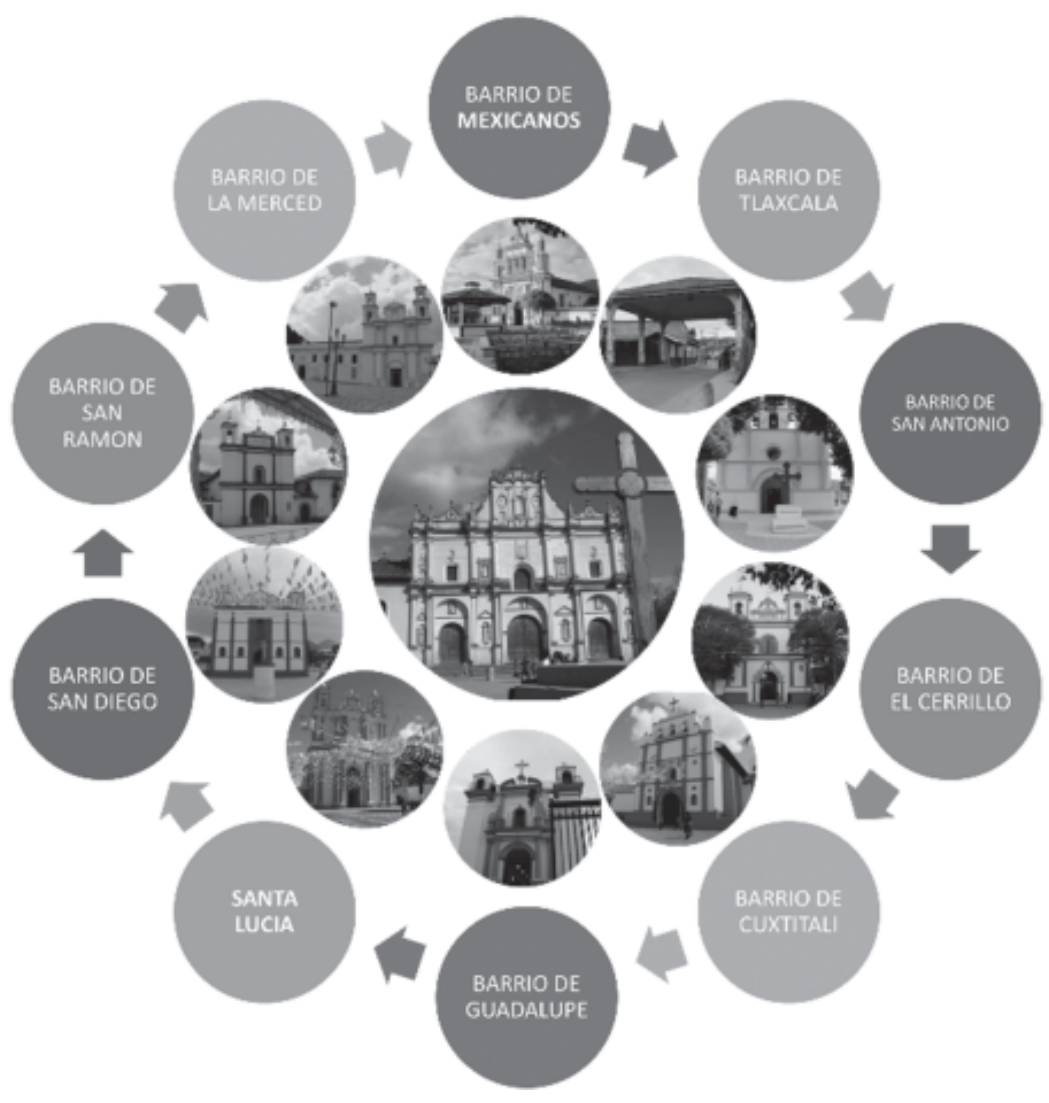

Fuente y elaboración propia

Además de la diversidad de artesanía que se fabrica y comercializa en la ciudad, en el ámbito regional, las comunidades indígenas manufacturan principalmente artesanía textil que se borda de acuerdo a diversos patrones identitarios y se emplean para fabricar de diversas prendas como faldas, chales, sacos, abrigos y bolsas entre otras prendas y desarrollan además otros oficios tradicionales como el trabajo en hierro forjado, joyería y joyería de ámbar, alfarería, etc. Cada comunidad plasma su origen y su identidad con sus productos a partir de los diversos patrones, gráficos, geometrías y colores que son bordados en sus prendas y que son el elemento diferenciador entre cada una. Cada comunidad se representa a sí misma a través del arte textil comunicando y haciendo visible su origen y procedencia, y empleando colores y técnicas que son aprendidas y transmitidas de generación en generación. 
De acuerdo a lo anterior en el ámbito local y regional, existen importantes recursos tangibles e intangibles manifestados por una gran variedad de productos culturales, los cuales son susceptibles de comercializarse generando recursos económicos de apoyo a su economía. En el siguiente esquema explicaremos cuales son los recursos y productos que representan el insumo principal que puede contribuir a potencializar la economía creativa de la ciudad y la región y el fomento a su propia economía, y teniendo esto en cuenta, alinear una estrategia para la vinculación a la red de ciudades creativas de la UNESCO y la forma de contribuir a los objetivos de dicha red.

Figura 7. Insumos creativos para el fomento de la economía creativa en San Cristóbal de Las Casas y la región.

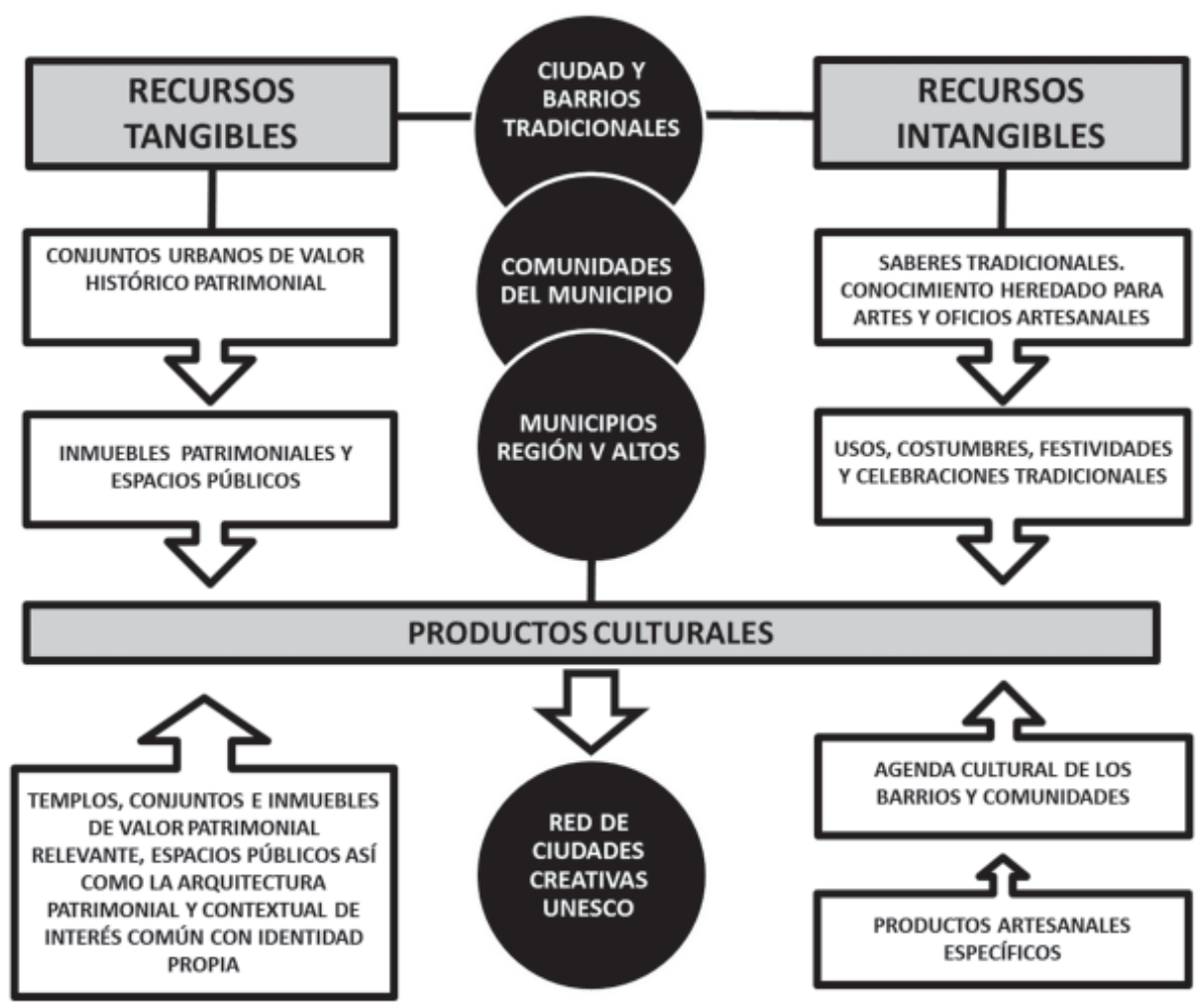

Fuente y elaboración propia 


\section{Recursos tangibles para el fomento a la economía}

En la ciudad y en los barrios el patrimonio arquitectónico es considerado como un importante recurso tangible, el cual está compuesto tanto por los inmuebles y conjuntos arquitectónicos de carácter relevante del barrio, principalmente el templo y la plaza, que en todos los casos es el hito de referencia y el elemento jerárquico, y que está acompañado por una gran cantidad de arquitectura vernácula de valor contextual, lo que le otorga un carácter único y especial a la ciudad y a cada barrio en específico. De esta forma, el espacio urbano por sus características y sus elementos componentes, son uno de los principales recursos que atraen al turismo y a los visitantes, en conjunto con la gran cantidad de espacios públicos con que cuenta la ciudad. A su vez, estos espacios públicos sirven como protagonistas para el encuentro de los habitantes y visitantes, y como escenario para los diversos festejos y celebraciones tradicionales de cada barrio y la ciudad, sin contar su papel para el intercambio y comercialización de diversos productos culturales. Esto mismo sucede en las comunidades de la región, donde cada una por lo general cuenta con una estructura y composición urbana específica y en donde el espacio público es fundamental puesto que cumple las mismas funciones que en el ámbito local.

Figura 8. El barrio La Merced con su arquitectura y espacio público característico.

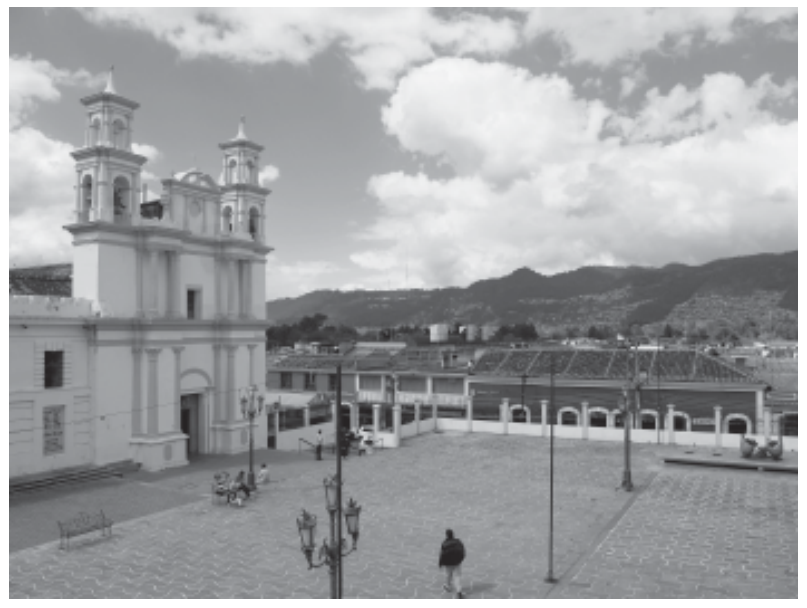

Fuente: Documento fotográfico de Mónica Solórzano
Figura 9. El barrio de Santa Lucía.

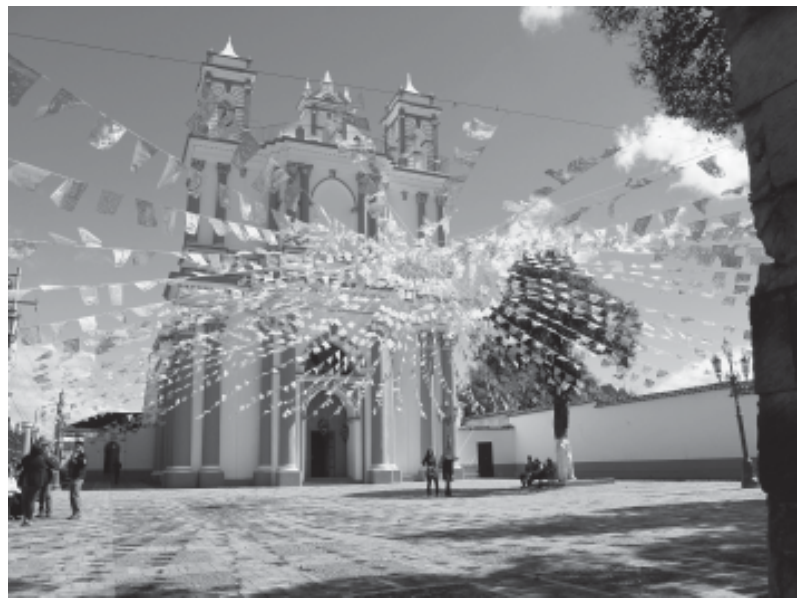

Fuente: Documento fotográfico de Mónica Solórzano

\section{Recursos intangibles para el fomento a la economía}

Además de los recursos tangibles mencionados anteriormente, cada barrio, comunidad o municipio de la región cuenta con otros recursos intangibles igualmente valiosos, los cuales están representados fundamentalmente por dos aspectos: 
(a) los usos, costumbres, festividades y celebraciones tradicionales, y

(b) los saberes y oficios tradicionales que incluyen todos los conocimientos tradicionales heredados de generación en generación para la elaboración de las artes y oficios artesanales diversos.

Figura 10. Artesano alfarero del barrio de San Ramón.

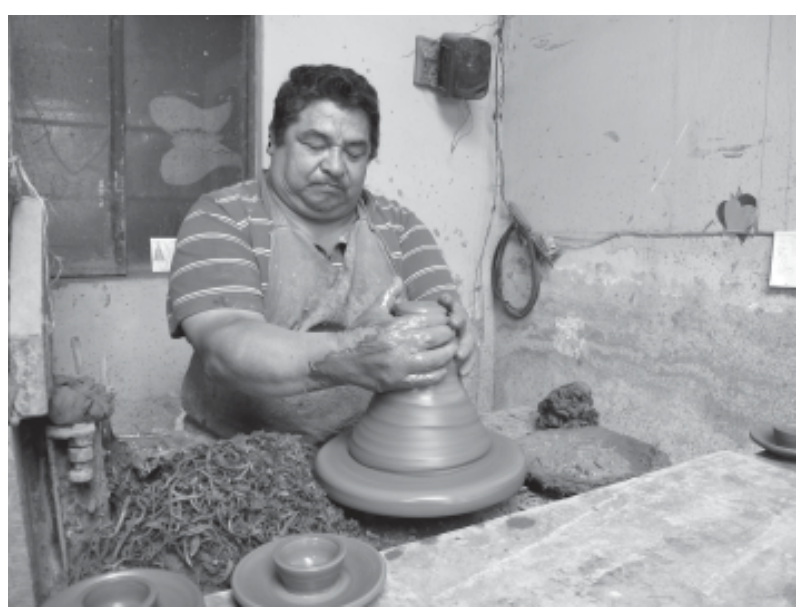

Fuente: Documento fotográfico de Mónica Solórzano
Figura 11. Artesano textil del barrio de mexicanos.

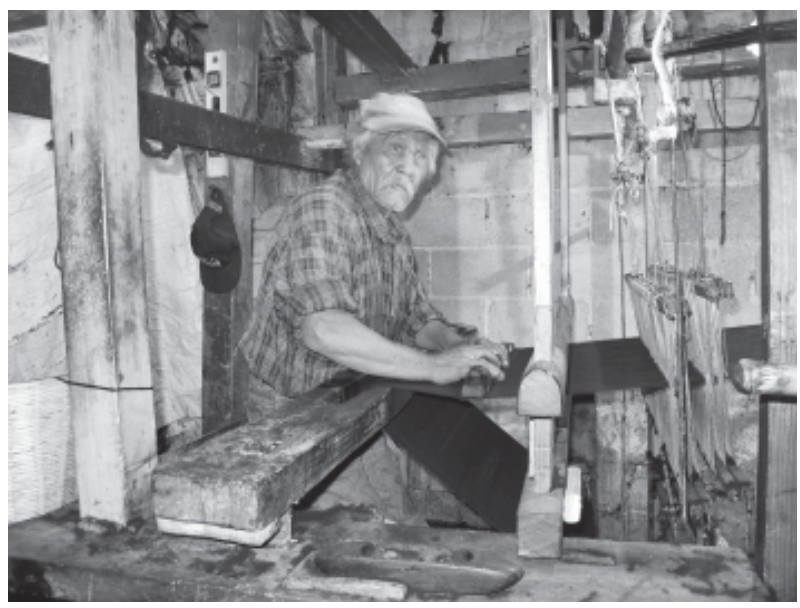

Fuente: Documento fotográfico de Mónica Solórzano

Los usos, costumbres, festividades y celebraciones en los barrios de San Cristóbal de Las Casas así como de los diversos municipios de Chiapas están llenas de color, folklore y tradición, y tienen una carga simbólica y significativa, importante por representar un conocimiento adquirido y transmitido de generación en generación y que lleva implícitas las características de cada lugar. Estos factores contribuyen a la riqueza de los oficios tradicionales y posibilita la fabricación de la gran variedad de artes y artesanía popular empleando técnicas y saberes que son heredados y transmitidos por sus ancestros con una importante carga identitaria. Cada festividad que se realiza en la ciudad y los barrios, lleva implícita las creencias e ideologías de la gente que habita los barrios y la ciudad y que contribuye a esta riqueza cultural como recurso intangible. Podemos ver en el calendario siguiente, como la ciudad está prácticamente vinculada a estos eventos que permiten compartir y transmitir sus costumbres y tradiciones tanto al habitante como al visitante, convirtiendo la ciudad en un elemento vivo y con una cantidad importante de actividades culturales diversas. 
Figura 12. Calendario de festividades y tradiciones de la ciudad y los barrios.

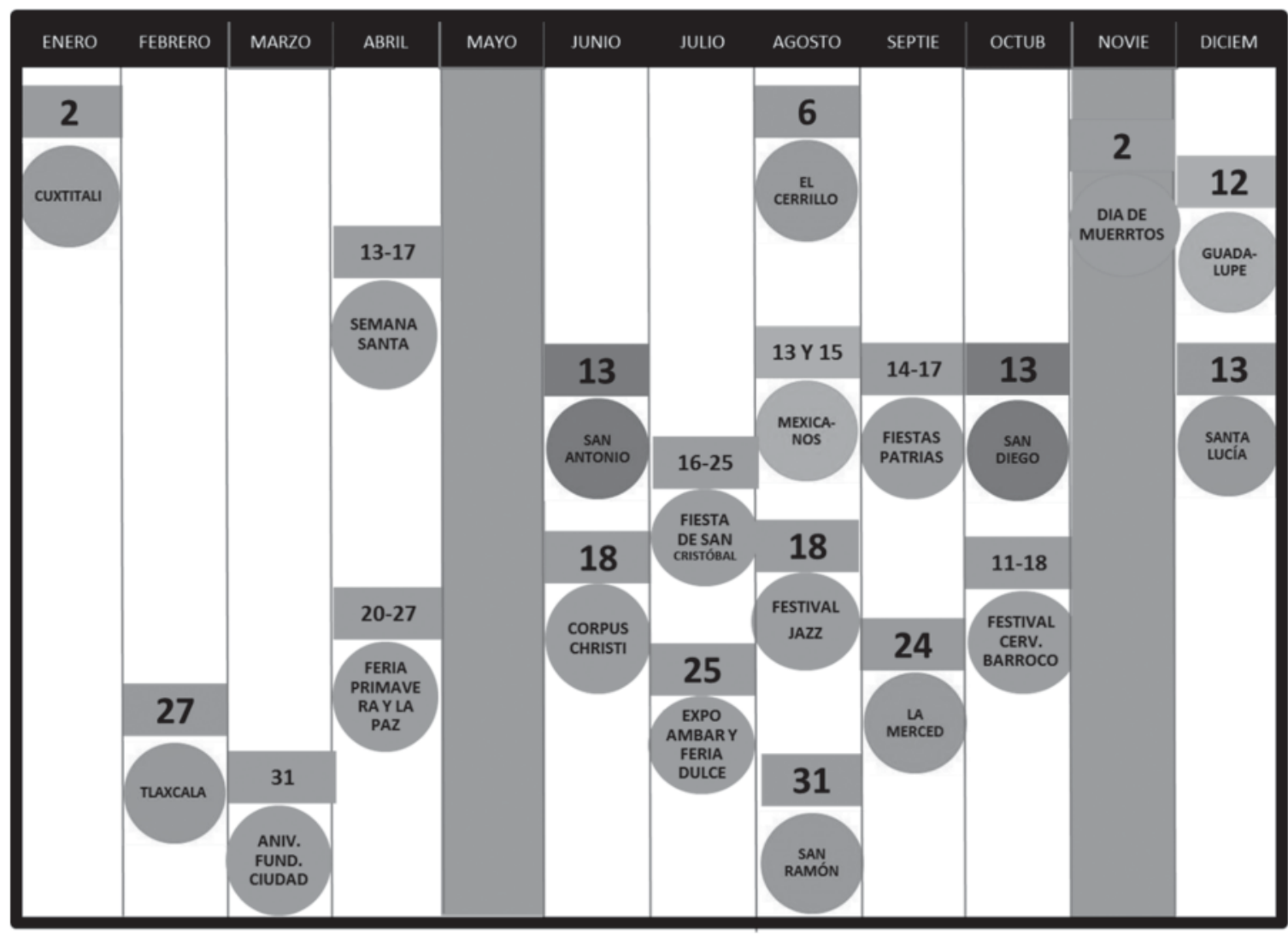

Fuente y elaboración propia

\section{Recursos que generan productos culturales de la ciudad y la región}

Los recursos tangibles e intangibles antes mencionados, contribuyen a generar una diversidad de productos culturales específicos que, si se alinean a las estrategias y políticas publicas adecuadas, pueden contribuir al fomento de la actividad económica no solo a los barrios de la ciudad y de las comunidades de la región, sino a todos en su conjunto, impulsando su desarrollo y generando beneficios económicos en torno a estos productos culturales. Como productos culturales, como elementos, objetos o eventos derivados de los diversos recursos tanto tangibles como intangibles se pueden considerar los siguientes:

\section{- La agenda cultural del barrio o de cada comunidad de la región}

Es la serie de eventos y festividades que se configura a partir de los diversas celebraciones tradicionales que se llevan a cabo, y que a su vez constituyen un factor para el fomento y preservación de su identidad. Es un elemento atractivo tanto para el habitante como para el visitante e incluyen varias actividades que abarcan diversos sectores creativos, como música, teatro, actividades culturales y artesanales, mercados 
de gastronomía tradicional, conciertos, desfiles de carros alegóricos así como eventos y festivales de música, entre otros. Además, organizados por la iniciativa privada, las ONG, y otros colectivos de la sociedad civil, de forma permanente y regular se realizan eventos y actividades culturales como conciertos, funciones de teatro, cine, exposiciones de arte en galerías y museos así como actividades y talleres artísticos y culturales. Estas actividades se considera que pueden contribuir en gran medida a generar impactos significativos de tipo económico, si se estructura una estrategia y políticas públicas del fomento y promoción adecuadas, incluyendo diversos públicos y actividades que impulsen la economía en torno a esto.

\section{- Los productos artesanales}

Productos artesanales específicos que producen en cada sitio, como elementos tangibles que derivan de un recurso intangible, y que regularmente se comercializan y se consumen tanto a nivel local y regional y que además, es uno de los principales productos que se comercializan a los turistas que regularmente visitan la ciudad. El aprovechamiento de estos productos artesanales, y el establecimiento de estrategias y políticas adecuadas de producción, promoción, comercialización de los diversos productos artesanales, debería garantizar que los beneficiarios directos sean los propios artesanos productores, y no los intermediarios que comercializan dichos productos. Asimismo, es indispensable realizar mapeos para cuantificar de forma real el impacto en el sector económico de esta actividad comercial, que en muchos de los casos, no figura dentro de los indicadores económicos oficiales. Hay un importante sector comercial que produce ingresos considerables a la ciudad, y que deriva de los productos culturales como la artesanía y arte popular, pero que no se cuantifica en los indicadores económicos oficiales. Se requiere alinear una estrategia para identificar el impacto real de este sector comercial de productos culturales.

Ambos tipos de productos culturales deben servir para generan recursos económicos que ayuden a impulsar de manera formal la economía local y regional, aprovechando de forma integral todos los recursos tangibles e intangibles con que cuentan tanto los barrios de la ciudad como las comunidades de la región, y alinear una política y estrategia específica para el impulso económico y el beneficio directo a los propios artesanos, creadores y sectores creativos. Más adelante se presenta un análisis general preliminar que nos ayuda a sustentar esta propuesta y considerarla como una posibilidad para el fomento a la economía a partir de la creatividad. 
Figura 13. Diversos productos artesanales elaborados en la ciudad y la región derivados de los recursos tangibles e intangibles con que se cuenta.

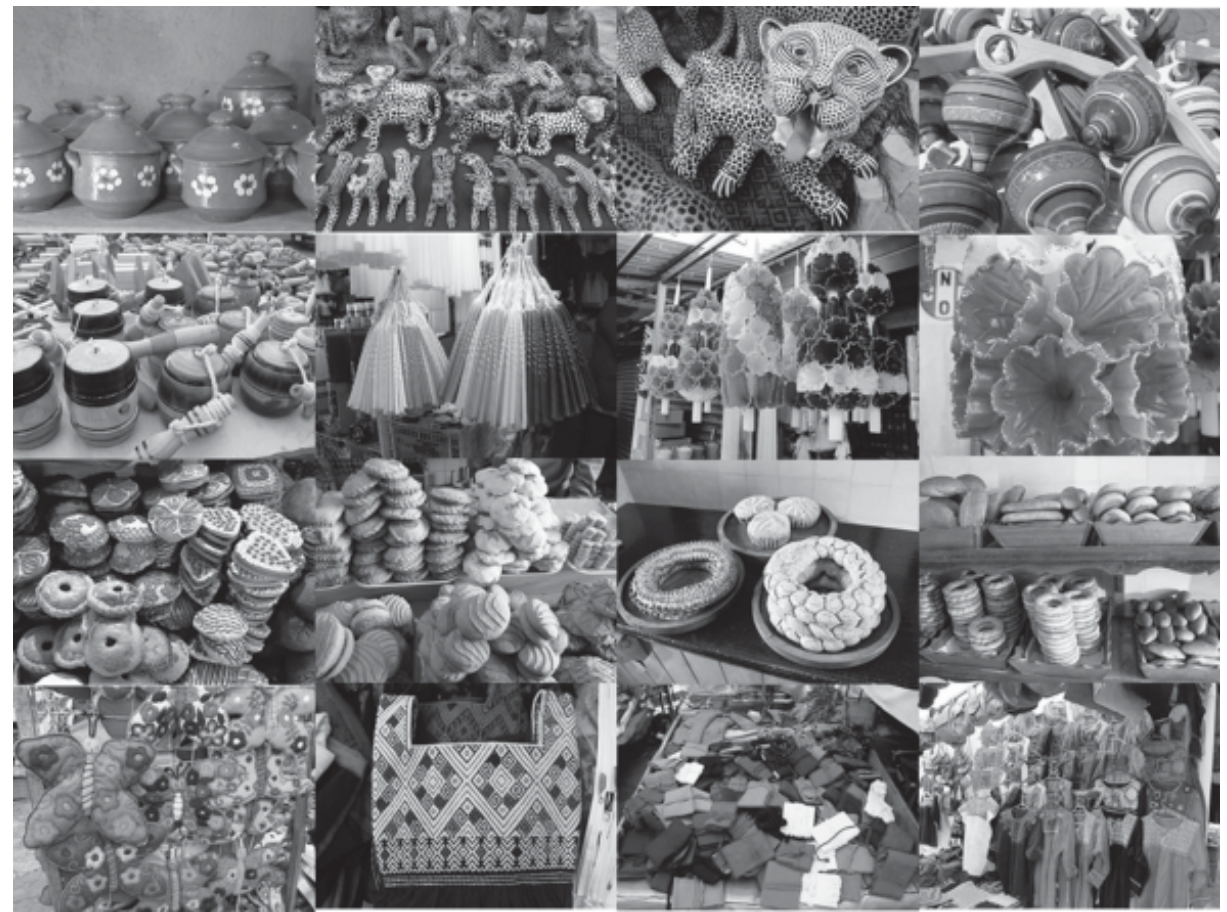

Fuente: Documento fotográfico de Mónica Solórzano

\section{Datos estadísticos sobre población y economía}

San Cristóbal de Las Casas es un municipio urbano rural con población intercultural en donde el idioma oficial es el español aunque se hablan diversas lenguas indígenas y cuenta mayoritariamente con población femenina. De acuerdo a datos oficiales generados por el Instituto Nacional de Estadísticas Geografía e Informática (INEGI), en el año 2010, San Cristóbal de Las Casas registró una población total de 185917 habitantes, de los cuales el $52.13 \%$ son mujeres y el $47.87 \%$ son hombres. La edad media es de 22 años, lo cual indica que es una población joven. La población de 50 años y más representa el $9.98 \%$ de la población total. Asimismo, del total de la población en edad de trabajar, el $40.05 \%$ es considerada población económicamente activa (PEA), lo que da un total de 74452 personas que trabajan o buscan trabajo en el municipio. De ellas, el 63.25\% son hombres y 36.75\% mujeres. En lo referente a la población ocupada y desocupada, cabe señalar que para el año 2010 la población económicamente activa desocupada, correspondía al 2.5\%, en tanto el $97.5 \%$ sí disponía de un empleo. Si bien se puede afirmar que la tasa de desempleo es baja, también se puede afirmar que el nivel de ingresos de la población es bajo, tomando en cuenta que por cada 100 personas que disponen de un empleo, 59 de ellas no tiene ingresos superiores a dos salarios mínimos mensuales. Es así que tan solo el 35.52\% de la población trabajadora percibe más de dos salarios mínimos al mes (Ayuntamiento de San Cristóbal de las Casas, s.f., p. 81). 
A su vez, la región Altos de Chiapas tiene una extensión territorial de 3717.08 km², y cuenta con una población de 601190 habitantes, de los cuales 290416 son mujeres y 310774 son hombres. La región tiene un índice de analfabetismo importante siendo del 26.8\% en donde solamente 15520 personas asisten a la escuela. Del total de la población solamente 193299 son población económicamente activa. Esta región está conformada por 17 municipios -en su gran mayoría indígena rural- que son: Aldama, Amatenango del Valle, Chalchihuitán, Chamula, Chanal, Chenalhó, Huixtán, Larráinzar, Mitontic, Oxchuc, Pantelhó, San Cristóbal de las Casas, San Juan Cancuc, Santiago El Pinar, Tenejapa, Teopisca y Zinacantán (Secretaría de Hacienda, Gobierno del Estado de Chiapas, s.f.).

Sobre la actividad económica de la ciudad y la región, además del turismo que es el sector que genera impactos económicos importantes en la ciudad, es interesante destacar que a raíz del movimiento armado de 1994, surgieron en la ciudad una gran cantidad de asociaciones civiles, colectivos y organizaciones que trabajan para lograr diversos objetivos sociales a favor de los grupos indígenas de la región. Muchas de estas ONG fomentan los oficios tradicionales de las comunidades de la región para la creación de las artesanías y arte popular como estrategia para favorecer el empleo y la prosperidad de dichas comunidades. Estas organizaciones, en conjunto con los artesanos y artesanas, promueven el diseño de nuevos productos con criterios de innovación, tomando como base el empleo de procesos y técnicas artesanales tradicionales, desarrollando así diversos productos de la mano de los artesanos de la región. Por lo tanto, el sector comercial de productos y servicios artesanales, tiene una demanda importante tanto para el habitante pero sobre todo para el visitante.

Figura 14. Algunos de los Colectivos, las ONG e instituciones que fomentan la economía creativa.

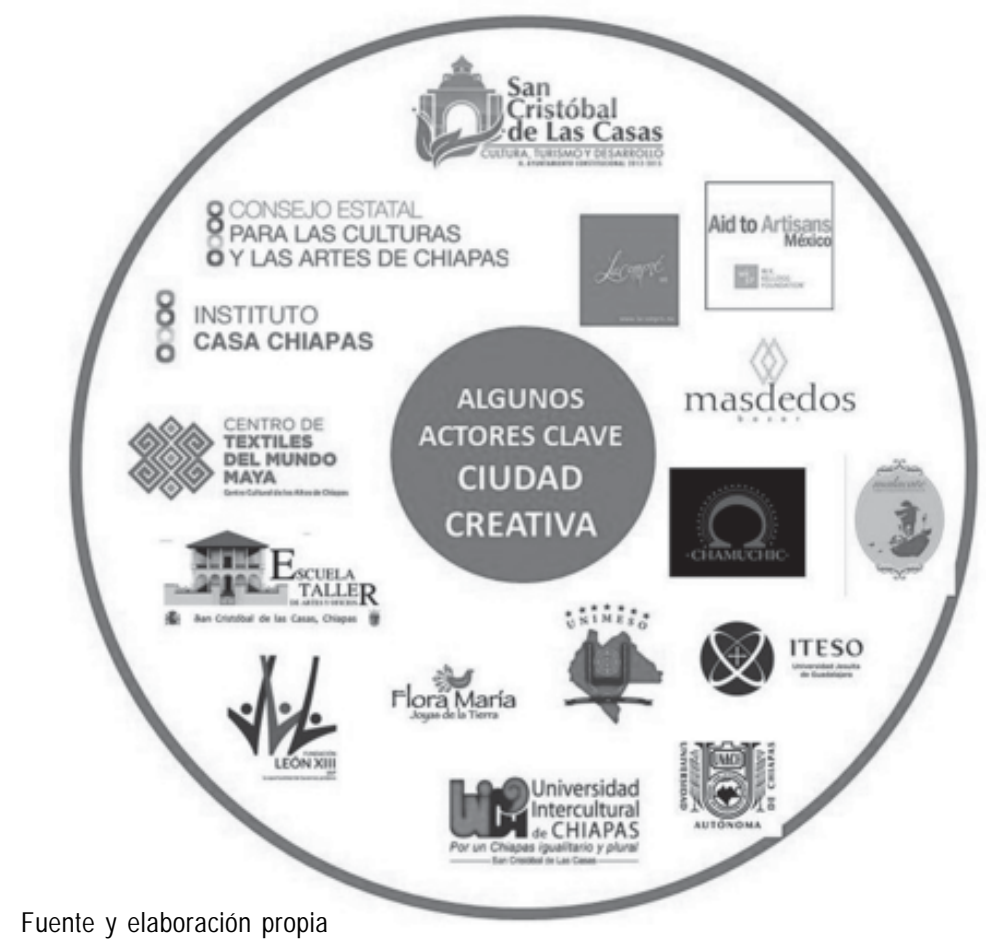


Muchas de estas organizaciones que trabajan el sector artesanal específicamente, se constituyen como emprendimientos creativos con objetivos sociales, y buscan beneficiar a diversas comunidades a partir de su propia creatividad. Este hecho particular ya ha generado un mercado importante de productos y servicios artesanales en la ciudad, integrando una carga interesante de innovación. Muchos de estos productos que combinan tradición con innovación y diseño ya se comercializan con mucho éxito en la ciudad y la región, es de considerar que estas organizaciones trabajan de forma continua, de la mano de los grupos indígenas en la generación de diversos productos y generando empleos y fuentes de ingreso para las familias de artesanos de las comunidades cercanas y de la región. Estas organizaciones civiles como tal tienen ya un papel importante y fundamental para la puesta en valor y fomento del trabajo artesanal, tanto para la población local, como para el turismo que visita la ciudad. Entre la variedad de productos innovadores que generan están los textiles y bordados y a través de ellos la confección de diversas prendas y accesorios para toda la familia, zapatos, productos para hogar y decoración como mantelería, tapetes, etc., así como la fabricación joyería artesanal empleando materia prima del lugar y la promoción de productos gastronómicos y alimentarios innovadores con técnicas tradicionales, entre los que destaca el mercado creciente del café y sus diversas mezclas, entre otros muchos productos incentivados en gran medida, por el trabajo de las organizaciones civiles. Estos grupos de ONG y colectivos han generado muchos beneficios a diversas comunidades del municipio y de la región, así como a los artesanos y artesanas de los propios barrios de la ciudad.

Figura 15. Metodología empleada para el mapeo preliminar realizado con algunos emprendedores de la ciudad para identificar el impacto de la creatividad en la economía.

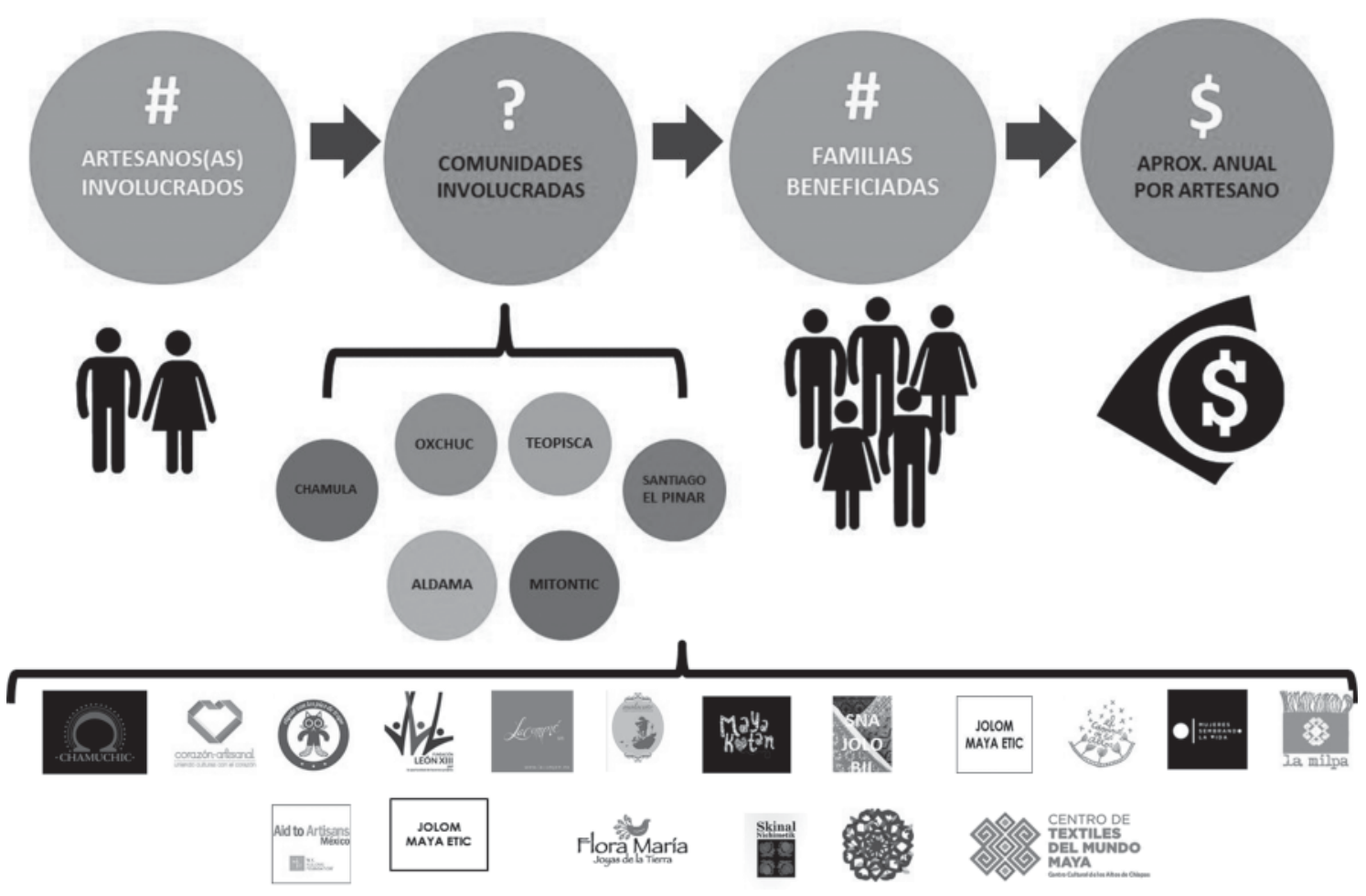

Fuente y elaboración propia 
En un ejercicio de mapeo preliminar realizado por la autora, identificando a más de 25 emprendimientos creativos que trabajan con artesanos de las comunidades de la región y de los barrios de la ciudad se pudieron encontrar algunos indicadores interesantes. Los datos obtenidos al analizar la información de tan solo de 14 de estos emprendimientos (del resto no se tuvo acceso a cifras y datos específicos), indican que se generan beneficios directos a más de 2000 artesanos en las distintas comunidades. Esto se traduce en más de 1000 empleos directos de artesanos y artesanas en 14 de los 17 municipios de la región incluido San Cristóbal de Las Casas.

Tabla 2

Datos estimados de ejercicio de mapeo preliminar para visualizar el impacto de la creatividad en la economía de San Cristóbal de Las Casas

\begin{tabular}{|c|c|c|c|c|}
\hline $\begin{array}{c}\text { ONG / ORGANIZACIÓN / } \\
\text { COLECTIVO / } \\
\text { EMPRENDEDOR CREATIVO } \\
\text { (datos estimados) }\end{array}$ & \# artesanas/os & $\begin{array}{c}\text { Municipios } \\
\text { involucrados }\end{array}$ & $\begin{array}{l}\text { Comunidades } \\
\text { involucradas }\end{array}$ & $\begin{array}{l}\text { Empleos } \\
\text { directos que } \\
\text { genera }\end{array}$ \\
\hline Aid to artisans. & 400 & 11 & $\mathrm{~s} / \mathrm{d}$ & $\mathrm{s} / \mathrm{d}$ \\
\hline Chamuchic & 20 & 3 & $\mathrm{~s} / \mathrm{d}$ & 4 \\
\hline Corazón artesanal & 27 & 3 & $s / d$ & $s / d$ \\
\hline El gato con los pies de trapo & 11 & 1 & $s / d$ & 13 \\
\hline Fundación León XIII & 425 & 12 & $s / d$ & 5 \\
\hline K'un K'un Talleres de artes y oficios & 20 & 2 & $\mathrm{~s} / \mathrm{d}$ & 20 \\
\hline Lacompré & 36 & 2 & $\mathrm{~s} / \mathrm{d}$ & 14 \\
\hline Malacate taller experimental textil & 20 & 6 & $\mathrm{~s} / \mathrm{d}$ & 5 \\
\hline Maya Kotan. & 12 & 1 & $\mathrm{~s} / \mathrm{d}$ & 7 \\
\hline Sna Jolobil. & 800 & $s / d$ & 30 & $s / d$ \\
\hline Jolom Maya etic. & 250 & $\mathrm{~s} / \mathrm{d}$ & $s / d$ & $s / d$ \\
\hline El camino de los Altos. & 130 & 5 & $s / d$ & $\mathrm{~s} / \mathrm{d}$ \\
\hline Casa Chiapas & 350 & 14 & $\mathrm{~s} / \mathrm{d}$ & 984 \\
\hline Flora Maria & 10 & 1 & $s / d$ & 6 \\
\hline TOTAL & 2511 & 61 & 30 & 1058 \\
\hline
\end{tabular}

Fuente y elaboración propia

Nota: $s / d=\sin$ datos 
Aun cuando esto es un ejercicio de valoración preliminar realizado con una parte de las ONG cuantificadas, los datos anteriores muestran de forma parcial, el gran impacto económico para la ciudad y la región de solo algunos de los muchos emprendimientos creativos que existen y operan ya en San Cristóbal. Es de llamar la atención que el impacto real en términos económicos del sector creativo no está cuantificado en los indicadores oficiales, ni forman parte las cifras e indicadores que presentan las entidades oficiales sobre la economía de la ciudad. Sin duda es un reto el incluir a la cultura dentro de los indicadores económicos, y las cifras anteriores solo una muestra el gran potencial que tiene el sector creativo, y que como tal, ya está funcionado como motor de desarrollo para un importante grupo de artesanos y creativos. Se requiere por tanto, como reto principal, reconocer este potencial y aprovecharlo para ser impulsado de forma oficial como uno de motores de desarrollo, alineando con ello diversas políticas públicas y estrategias de impulso y fomento al sector artesanal y a las industrias creativas, además del sector turístico, que por sí mismo es uno de las más importantes fuentes de ingreso de la ciudad. La vinculación de sectores turístico y creativo, como motor de desarrollo aprovechando el potencial de la ciudad, sin duda representaría un importante motor de desarrollo para la ciudad, aprovechando sus recursos de forma sustentable, y con ello, contribuyendo a conservar y preservar sus tradiciones y valores patrimoniales.

\section{Conclusiones}

El proyecto de Ciudad Creativa presentado por San Cristóbal de las Casas tiene varias características innovadoras, al ser la primera ciudad en México en considerar la economía creativa como alternativa para su desarrollo buscando un cambio de paradigma para generar escenarios que garanticen la prosperidad de la ciudad y de las comunidades de una de las regiones más pobres de México.

Este proyecto ha contribuido a reconocer el potencial para la creación de redes locales, regionales e internacionales de vinculación creativa y el fortalecimiento de las capacidades en diversos niveles para trabajar con aspectos de innovación basados en la consideración a su propia tradición.

El reto más importante es la comprensión por parte de los diversos grupos políticos y sociales, del potencial y compromiso que adquiere la ciudad como impulsor del cambio y desarrollo regional a través de la economía creativa. Asimismo, la ciudad asume diversos retos que se derivan de los ámbitos o esferas de acción en las que se involucra desde lo local y sus barrios tradicionales, la estrecha vinculación de la ciudad y los 17 municipios de la Región Altos de Chiapas, en su mayoría indígenas y aprovechar todo ello para establecer alianzas e intercambios creativos a nivel internacional con otras ciudades de la red. Sin duda, se requiere alinear estrategias y políticas públicas, para aprovechar la creatividad existente y toda la riqueza cultural de la ciudad y región, como motor de desarrollo. Esto creemos es posible, sin duda representa es un gran reto para México, y la ciudad de San Cristóbal de Las Casas, se aventura esta tarea que se antoja difícil, pero que bien vale la pena el aprovechar la oportunidad de intentarlo. 


\section{Referencias}

Aubry, A. (2008). San Cristóbal de Las Casas: Su Historia Urbana, Demografía y Monumental 1528-1990. Chiapas: ADABI.

Ayuntamiento de San Cristóbal de las Casas (s.f.). Plan de Desarrollo Municipal 2012-2015. Recuperado de http://sancristobal.gob.mx/ download/PLANASUBIR.pdf

Buitrago, F., \& Duque, I. (2013). La economía naranja, una oportunidad infinita. Washington: BID.

Consejo Nacional de Evaluación de la Política de Desarrollo Social - CONEVAL (2011). Medición de la pobreza en los municipios de México 2010. Recuperado de http://www.coneval.gob.mx/informes/Pobreza/Pobreza_municipal/Presentacion/Pobreza_municipios.pdf

Fonseca, A. C. (2008). Economía creativa como Estrategia de Desarrollo - una visión de los países en desarrollo. Sao Paulo: Garimpo de Solucoes e Itaú Cultural.

Instituto Nacional de Estadística, Geográfica e Informática - INEGI (2010). Censo de población y vivienda 2010. Recuperado de http://www.inegi.org.mx/est/contenidos/proyectos/ccpv/cpv2010/Default.aspx

Secretaría de Hacienda, Gobierno del Estado de Chiapas (s.f.). Programa Regional de Desarrollo. Región V Altos Tsotsil - Tseltal. Recuperado de http://www.haciendachiapas.gob.mx/planeacion/Informacion/Desarrollo-Regional/prog-regionales/ALTOS.pdf

Solórzano, M. (2015). Ciudades creativas y patrimonio cultural: nuevos escenarios para la conservación del patrimonio en México. En F. J. López-Morales, \& F. Vidargas (eds.), Encuentro Internacional. Usos del patrimonio: Nuevos escenarios. México: Instituto Nacional de Antropología e Historia. Recuperado de https://rei.iteso.mx/handle/11117/3796

UNESCO (Agosto, 2015). Industrias creativas. Recuperado de http://www.unesco.org/new/es/santiago/culture/creative-industries/ 